\title{
Theoretical Analysis of Voltammetry at a Rotating Disk Electode in the Absence of Supporting Electrolyte
}

\author{
R. Saravanakumar ${ }^{\dagger}$, P. Pirabaharan ${ }^{\dagger}$, Marwan Abukhaled ${ }^{\ddagger}$ L. Rajendran ${ }^{\S}$ \\ ${ }^{\dagger}$ Department of Mathematics, University College of Engineering, Anna University, Dindigul, India \\ Department of Mathematics and Statistics, American University of Sharjah, UAE \\ $\S$ Department of Mathematics, AMET (Deemed to be university), Kanathur, Chennai, Ind ia
}

\section{Supporting information:}

Matlab program for the numerical solution of nonlinear differential equation (15).

function sol=ex2

ex2init=bvpinit(linspace $(0,5),[21])$;

sol=bvp4c(@ex2ode, @ex2bc,ex2init $)$

end

functiondydx=ex2ode $(x, y)$

$\mathrm{dydx}=[\mathrm{y}(2)$

$((5 * \mathrm{y}(2)) /(\mathrm{y}(1) * \mathrm{y}(1)))-3 * \mathrm{x} * \mathrm{x} * \mathrm{y}(2)] ;$

end

function res $=\operatorname{ex} 2 b c(y a, y b)$

res $=[y a(1)-2$

yb(1)-1];

end 